CASO CLÍNICO

\title{
Síndrome de Forestier: caso clínico y revisión de la literatura
}

\author{
Forestier syndrome: clinical case and literature review \\ Luisa Palma, ${ }^{1}$ Javier Molina. ${ }^{2}$ \\ 'Médica Residente I año, Postgrado en Medicina de Rehabilitación, Facultad de Ciencias Médicas, UNAH; \\ ${ }^{2}$ Médico Especialista en Fisiatría; Unidad de Medicina Física y Rehabilitación IHSS Barrio Abajo.
}

RESUMEN. Antecedentes: El síndrome de Forestier también conocido como Hiperostosis Esquelética Idiopática Difusa (DISH, por sus siglas en inglés), es una enfermedad de etiología desconocida que se caracteriza por osificación del ligamento espinal anterior, siendo las porciones cervicales y torácicas las que se afectan más frecuentemente. Esta enfermedad es más frecuente en hombres y se asocia con diabetes, hipertensión arterial, dislipidemia y trastornos endocrinos. Descripción del caso clínico: Paciente femenina de 63 años con antecedente de dolor cervical desde hace 32 años, que 6 años después del inicio del cuadro, presentó limitación en la movilidad del cuello; presentando varios episodios de disfonía desde hace 10 años; al momento de la consulta la paciente presentó limitación de la movilidad del cuello y dolor cervical. La imagen de resonancia magnética reportó: presencia de crecimiento óseo anterior de los cuerpos vertebrales, este hallazgo está en relación con el síndrome de Forestier. Conclusiones: Por ser una enfermedad poco conocida es subdiagnosticada y a menudo confundida con otras patologías. Los pacientes son diagnosticados muchos años después de que aparecieron los primeros síntomas que incluyen dolor, limitación de la movilidad, disfagia y dificultad respiratoria. El tratamiento incluye manejo sintomático, terapia física y manejo quirúrgico.

Palabras clave: Columna vertebral, Hiperostosis esquelética difusa idiopática, Tratamiento conservador.

Recibido: 20-02-2020 Aceptado para publicación 16-02-2021

Dirección para correspondencia: Dra. Luisa Palma

Correo electrónico: luisapalmasie@gmail.com

Declaración de relaciones y actividades financieras y no financieras y conflictos de interés: ninguno.

\section{INTRODUCCIÓN}

El Síndrome de Forestier fue conocido por varias décadas como hiperostosis anquilosante senil de la columna, Forestier y Rotes Querol en 1950, la describen como hiperostosis anquilosante difusa conocida por las siglas en ingles DISH (diffuse idiopathic skeletal hiperostosis). ${ }^{1,2}$ Esta enfermedad reduce la calidad de vida y la autosuficiencia de los pacientes. La característica de esta enfermedad es la osificación de los ligamentos y las entesis en las regiones prevertebral y prediscal de la columna vertebral. La calcificación hiperostótica es más frecuente en los ligamentos anterolaterales de las vértebras torácicas. Sin embargo, las vértebras cervicales y lumbares también pueden afectarse. ${ }^{2}$

La prevalencia oscila entre el 2.9 y el $42 \%$, dependiendo de la diversidad racial y étnica y de las variaciones en el tipo de imagen y los criterios de clasificación utilizados. Es más común en hombres que en mujeres. ${ }^{3,4}$

La etiología es desconocida, pero se asocia con la diabetes mellitus tipo 2, hipertensión arterial, edad avanzada, síndrome metabólico, obesidad, y otras enfermedades cardiovasculares. A nivel molecular, está relacionada con factores genéticos, metabólicos, mecánicos, vasculares, con cambios en las vías de señalización y aumentos de los factores de crecimiento. ${ }^{5,1}$ Los estudios realizados en cadáveres sugieren un papel limitado de la degeneración del disco intervertebral en la patogénesis. ${ }^{6}$

En las etapas tempranas de la enfermedad la mayoría de pacientes suelen ser asintomáticos, la osificación en las imágenes del ligamento longitudinal cervical anterior es un hallazgo incidental, las manifestaciones son progresivas y evolucionan lentamente, entre las que se describen dolor torácico, lumbar y/o cervical, restricción de la movilidad, radiculopatía, dolor poliarticular, cefalea, sinovitis monoarticular, sensación de cuerpo extraño, disfonía, y un aumento del riesgo de fractura vertebral., ${ }^{2,7}$ Los depósitos óseos en la columna cervical pueden causar disfagia, aspiración, regurgitación, apnea del sueño, infección del tracto respiratorio superior, obstrucción de las vías respiratorias y una intubación difícil. ${ }^{8}$ La disfagia parece ser la presentación más común en los pacientes que presentan la enfermedad a nivel cervical, aunque sólo del 0.1\%-6\% desarrolla disfagia debido a los osteofitos cervicales. ${ }^{9,10}$ Debido 
a que es una enfermedad poco frecuente los autores consideran que es importante aportar conocimientos, siendo necesario la descripción de casos que permitan conocer la evolución clínica y opciones de manejo conservador disponibles.

\section{DESCRIPCIÓN DEL CASO CLÍNICO}

Se trata de una paciente femenina de 63 años, casada, originaria de Tegucigalpa, con antecedentes de hipertensión arterial diagnosticada hace 32 años, tratada con: carvedilol, candesartan y amlodipina; diabetes mellitus diagnosticada hacía 12 años, manejada con insulina NPH y cristalina.

Paciente con cuadro de cervicalgia desde el año 1982, tipo sordo, de moderada a severa intensidad, agravándose con el movimiento del cuello y el estrés emocional, atenuándose con analgésicos y relajantes musculares. Seis años después presento limitación de los rangos de movilidad articular del cuello, llevándola a realizar movimientos en bloque (cervical y torso) al realizar las rotaciones cervicales. Del año 2010 a 2020, presento 3 episodios de disfonía con duración de 8 a 15 días (2010, 2017, 2019). El 2015 refirió parestesias en ambos miembros superiores. En octubre del 2019 manifestó cervicalgia intensa y disfagia para sólidos por 15 días. La paciente presentaba limitaciones en las actividades de la vida diaria y restricciones en la participación; no podía conducir y se retiró de su trabajo.

A la exploración física realizada en enero 2020, se encontró sin facie característica, marcha independiente con pasos cortos, disminución del balanceo de los brazos. cuello simétrico, con inclinación anterior, disminución de los rangos articulares cervicales; flexión: $20^{\circ}$, extensión: $10^{\circ}$, inclinación derecha e izquierda: $10^{\circ}, 8^{\circ}$ respectivamente, rotación derecha e izquierda: $20^{\circ} 40^{\circ}$, dolor al movimiento activo y pasivo, no presentó alteraciones de la sensibilidad. A la palpación cervical presentó espasmo cervical bilateral y dolor a la palpación en toda la región cervical.

Desde el año 1982 la paciente recibió tratamiento sintomático (con mejoría transitoria), se le indico radiografía de la columna cervical, y el médico tratante en ese momento refirió lo siguiente: "se observa leve aumento de la osificación, que no tiene significancia”.

Posteriormente y debido a que el cuadro de la paciente no presentó mejoría, el médico tratante en octubre del 2019, decide referirla al servicio de ortopedia del Instituto Hondureño de Seguridad Social (IHSS) para evaluación; se le indica Imagen de resonancia magnética (IRM), al día siguiente se comunican vía telefónica para que acuda al reumatólogo quien le informa que la IRM reporta la presencia de crecimiento óseo anterior de los cuerpos vertebrales y que este hallazgo está en relación con el síndrome de Forestier.

La resonancia magnética realizada el 12 de octubre del 2019, evidenció la presencia de crecimiento óseo anterior, des- 


\section{DISCUSIÓN}

A pesar de ser una patología descrita desde hace varias décadas, el síndrome de Forestier es una enfermedad de etiología no establecida, asociada con algunas patologías: diabetes mellitus, hipertensión, dislipidemia y enfermedades metabólicas, ${ }^{1,2}$ patologías que van en aumento por lo que es de esperarse mayor incidencia en los casos de síndrome de Forestier. Respecto a las manifestaciones clínicas se deben a la alteración de la biomecánica de las estructuras adyacentes, ${ }^{3}$ como se observa en el caso expuesto, los síntomas de la paciente han sido progresivos iniciando con cervicalgia, luego disfonía, parestesias en miembros superiores y disfagia, resultado de alteración en dos estructuras de la región cervical, esófago y tráquea.

El desplazamiento de la tráquea y el esófago podría explicar el mecanismo de la disfagia y la obstrucción de las vías respiratorias que presentan los pacientes en etapas avanzadas, en las lesiones de la columna cervical, la compresión directa de la faringe y el esófago por los osteofitos, las alteraciones de la inclinación normal de la epiglotis, inflamación de los tejidos, edema alrededor del esófago, y el espasmo de los músculos de la faringe. ${ }^{3}$

Actualmente se diagnostica en estadios avanzados 0 cuando se presentan complicaciones graves. ${ }^{11}$ En este caso, la paciente presento síntomas desde el año 1982 y fue diagnosticada 37 años después de manera accidental.

El diagnóstico se basa típicamente en técnicas de imagen radiológica o en evaluaciones anatómicas post-mortem, porque no se dispone de síntomas clínicos o pruebas de laboratorio específicas para este síndrome. ${ }^{1}$ Para el diagnóstico se utiliza la clasificación radiológica de Resnick. ${ }^{1,12}$ Esta clasificación consta de los siguientes criterios clínicos: osificación (calcificación del ligamento longitudinal anterior) a lo largo de la cara anterolateral de al menos 4 cuerpos vertebrales contiguos, preservación de la altura de los discos, ausencia de esclerosis marginal en los cuerpos vertebrales y ausencia de degeneración en las articulaciones interapofisiarias 0 en las sacroilíacas. ${ }^{1,12}$ En este caso clínico se encontró que la paciente cumplió los criterios antes mencionados.

El diagnóstico diferencial incluye una variedad de enfermedades que presentan características similares con el síndrome de Forestier como la espondilitis anquilosante, espondilosis deformante, degeneración de la columna vertebral, melorreostosis, fluorosis, osteomalacia, acromegalia, hipervitaminosis A, paquidermoperiostosis, hipofosfatemia, hipoparatiroidismo. ${ }^{2}$ También se incluyen patologías de las estructuras relacionadas con la columna cervical como los tumores orofaríngeos, abscesos retrofaríngeos, divertículo de Zenker, tumores esofágicos y otras masas cervicales. ${ }^{13} \mathrm{En}$ este caso, la ausencia de degeneración de discos intervertebrales, erosión y anquilosis ósea de las articulaciones sacroilíacas, fueron consideradas para excluir estas enfermedades.

Los estudios radiológicos usados son las radiografías, TAC e IRM. ${ }^{14,12}$ Siendo la IRM el estudio más específico para localizar el nivel afectado. Las características de imagen que definen este síndrome son los osteofitos que se forman a lo largo del ligamento longitudinal anterior en una orientación horizontal y principalmente en el lado derecho. ${ }^{15}$
Las opciones de manejo conservador para el síndrome de Forestier incluyen modificación del estilo de vida, terapia física, los antiinflamatorios no esteroideos (AINE) locales o sistémicos, también se indican medicamentos antirreflujo, relajantes musculares y sedantes. En caso de disfagia refractaria, se puede indicar la alimentación enteral y la gastrostomía. ${ }^{13}$ Se ha encontrado que los bifosfonatos pueden ser capaces de reducir la formación de osteofitos tanto en los modelos animales como en los humanos. ${ }^{15}$

El tratamiento quirúrgico está indicado cuando fracasa el tratamiento conservador, aumento de la disfagia con pérdida de peso importante y obstrucción de las vías respiratorias superiores. Se realiza una resección localizada y limitada de los osteofitos cervicales anteriores(osteofitectomía)..$^{913,16}$ La paciente del caso se trató con manejo sintomático, terapia física y se planteó la posibilidad de intervención quirúrgica.

La asociación a los trastornos metabólicos se debe al aumento de factores de crecimiento que estos producen, sobre todo el factor de crecimiento similar a la insulina tipo 1 , aumentos de los mediadores inflamatorios: interleucina-1, interleucina-6 que activan el receptor de factor nuclear kappa-B, la proliferación de osteoblastos y finalmente la osificación. ${ }^{5,1}$

Se asocia con la hipertensión porque esta puede ser un estímulo para el desarrollo de daño temprano de las células endoteliales, aumentando el factor de crecimiento derivado de las plaquetas que puede generar proliferación de osteoblastos. ${ }^{1}$

El método de imagen que se usó para el diagnóstico del caso fue la IRM que es el método específico para definir el nivel de afectación. El tratamiento es sintomático. ${ }^{13}$ También se debe controlar las patologías asociadas que el paciente presente. En nuestro caso a la paciente se le indico tratamiento sintomático, terapia física y estar en control de sus patologías de base.

\section{CONCLUSIÓN}

Esta es una patología poco frecuente, de etiología desconocida, caracterizada por el crecimiento óseo en el ligamento longitudinal cervical anterior, los pacientes son diagnosticados accidentalmente cuando la enfermedad ha progresado significativamente como para producir síntomas severos y afectar la calidad de vida de los pacientes. En el caso descrito, los factores de riesgo de la paciente son diabetes mellitus e hipertensión arterial. La paciente del caso presentó varios de estos síntomas: dolor, limitación en los rangos de movimiento, parestesias en miembros superiores, disfonía y disfagia. Fue diagnosticada incidentalmente después de muchos años de síntomas y manejo por varios especialistas.

\section{CONTRIBUCIONES}

LP y JM participaron de manera conjunta en la preparación, redacción del presente manuscrito, aprobando su versión final.

\section{AGRADECIMIENTO}

A la Dra. Sonia Flores jefa de la Unidad de Medicina Física y Rehabilitación IHSS Barrio Abajo. 


\section{REFERENCIAS}

1. Kuperus JS, de Gendt EEA, Oner FC, de Jong PA, Buckens SCFM, van der Merwe AE, et al. Classification criteria for diffuse idiopathic skeletal hyperostosis: A lack of consensus. Rheumatology (Oxford). 2017;56(7):112334.

2. Vaishya R, Vijay V, Nwagbara IC, Agarwal AK. Diffuse idiopathic skeletal hyperostosis (DISH) -:A common but less known cause of back pain. J Clin Orthop Trauma [Internet]. 2017[consultado 22 mayo 2020];8(2):191-6. Disponible en:: http://dx.doi.org/10.1016/j.jcot.2016.11.006

3. Candelario N, Lo KB, Naranjo M. Cervical diffuse idiopathic skeletal hyperostosis (DISH) causing oropharyngeal dysphagia. BMJ Case Rep. 2017;2017:bcr2016218630.

4. Milner GR, Boldsen JL, Ousley SD, Getz SM, Weise S, Tarp P, et al. Selective mortality in middle-aged American women with Diffuse Idiopathic Skeletal Hyperostosis (DISH). PLoS One. 2018;13(8):1-14.

5. Torres Ávila I. Evidence from occupational therapy treatment on daily living activities in patients with stroke. Rev electrónica Ter Ocup Galicia, TOG [Internet]. 2014[consultado 22 mayo 2020];11(19):3. Disponible en: http:// dialnet.unirioja.es/servlet/articulo?codigo $=4710575 \&$ info=resumen\&idiom $a=E N G$

6. Kuperus JS, Westerveld LA, Rutges JPHJ, Alblas J, van Rijen MHP, Bleys RLAW, et al. Histological characteristics of diffuse idiopathic skeletal hyperostosis. J Orthop Res. 2017;35(1):140-6.

7. Holgate RLV, Steyn M. Diffuse idiopathic skeletal hyperostosis: Diagnostic, clinical, and paleopathological considerations. Clin Anat. 2016;29(7):8707.

8. Bakker JT, Kuperus JS, Kuijf HJ, Oner FC, De Jong PA, Verlaan JJ. Morphological characteristics of diffuse idiopathic skeletal hyperostosis in the cervical spine. PLoS One. 2017;12(11):1-11.

9. Yoshioka K, Murakami H, Demura S, Kato S, Yonezawa N, Takahashi N, et al. Surgical treatment for cervical diffuse idiopathic skeletal hyperostosis as a cause of dysphagia. Spine Surg Relat Res. 2018;2(3):197-201.

10. García García M. Forestier-Rotés Querol Disease: Cervical Radiological Progression and Onset of Dysphagia. Reumatol Clín. 2016;12(5):292-3.

11. Clavaguera $T$, Reyner $P$, Valls $R$, de Cendra $E$, Rodríguez-Jimeno MC. Diffuse idiopathic skeletal hyperostosis: Can we identify a peripheral pattern? Reumatol Clin [Internet]. 2019[consultado 22 mayo 2020];15(5):3014. Disponible en: http://dx.doi.org/10.1016/j.reuma.2017.03.015

12. Kim BS, Moon MS, Yoon MG, Kim ST, Kim SJ, Kim MS, et al. Prevalence of diffuse idiopathic skeletal hyperostosis diagnosed by whole spine computed tomography: A preliminary study. Clin Orthop Surg. 2018;10(1):41-6.

13. Ghammam M, Houas J, Bellakhdher M, Abdelkefi M. Dysphagia revealing diffuse idiopathic skeletal hyperostosis: Report of two cases and literature review. Pan Afr Med J. 2019;32:1-5.

14. Hirasawa A, Wakao N, Kamiya M, Takeuchi M, Kawanami K, Murotani K, et al. The prevalence of diffuse idiopathic skeletal hyperostosis in Japan: the first report of measurement by CT and review of the literature. J Orthop Sci [Internet]. 2016[consultado 22 mayo 2020]6;21(3):287-90. Disponible en: http://dx.doi.org/10.1016/j.jos.2016.02.001

15. Mader R, Verlaan JJ, Eshed I, Jacome BA, Puttini PS, Atzeni F, et al. Diffuse idiopathic skeletal hyperostosis (DISH): Where we are now and where to go next. RMD Open. 2017;3(1):1-6.

16. Press D. Progressive dysphagia and neck pain due to diffuse idiopathic skeletal hyperostosis of the cervical spine : a case report and literature review. Clin Interv Aging. 2014; 9: 553-557.

ABSTRACT. Background: Forestier syndrome, also known as Diffuse Idiopathic Skeletal Hyperostosis (DISH), is a disease of unknown etiology that is characterized by ossification of the anterior spinal ligament, being the cervical and thoracic portions the most affected frequently. This disease is more common in men and is associated with diabetes, high blood pressure, dyslipidemia, and endocrine disorders. Description of the clinical case: 63 -year-old female patient with a history of cervical pain for 32 years, who 6 years after the onset of the symptoms, presented limitation in neck mobility; she presenting several episodes of dysphonia for 10 years; At the time of consultation, the patient presented limited mobility of the neck and neck pain. The magnetic resonance image reported: presence of anterior bone growth of the vertebral bodies and that this finding is related to Forestier syndrome. Conclusions: Because it is a little-known disease, it is underdiagnosed and is often confused with other pathologies. Patients are diagnosed many years after the first symptoms appear, including pain, limited mobility, dysphagia, and respiratory distress. Treatment includes symptomatic management, physical therapy, and surgical management.

Keywords: Conservative treatment, Idiopathic diffuse skeletal hyperostosis, Spine. 